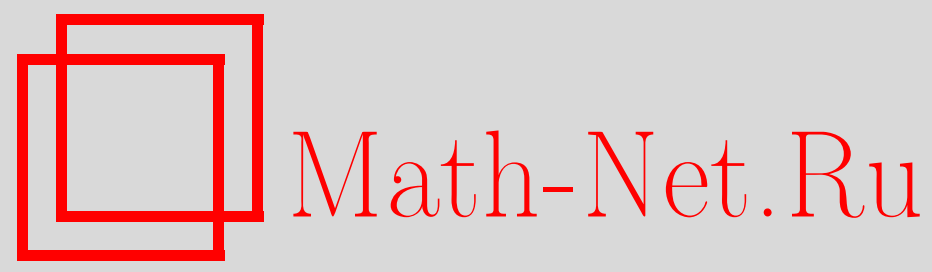

А. А. Карацуба, Омега-теоремы для дзетовых сумм, Мaтем. заметки, 2003, том 73, выпуск 2, 228-233

DOI: https://doi.org/10.4213/mzm181

Использование Общероссийского математического портала Math-Net.Ru подразумевает, что вы прочитали и согласны с пользовательским соглашением http://www .mathnet.ru/rus/agreement

Параметры загрузки:

IP : 52.6 .47 .48

26 апреля 2023 г., 15:40:45

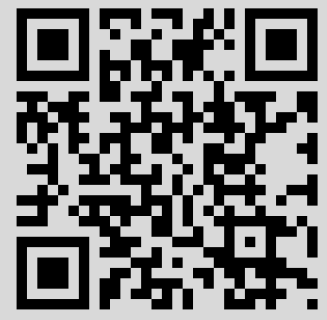




\title{
ОМЕГА-ТЕОРЕМЫ ДЛЯ ДЗЕТОВЫХ СУММ
}

\section{А. А. Карацуба}

\begin{abstract}
Получены нижние оценки модулей тригонометрических сумм теории дзета-функции Римана.

Библиография: 9 названий.
\end{abstract}

Будем употреблять устоявшиеся обозначения; в частности, $\zeta(s)$ - дзета-функция Римана, $s=\sigma+i t, i^{2}=-1, \sigma, t$ - вещественные числа; $c, c_{1}, \ldots$ - абсолютные положительные постоянные, в разных утверждениях, вообще говоря, разные; постоянные в знаках « абсолютные.

Дзетовыми суммами назьваются тригонометрические суммы $S$ вида (см. $[1$, с. 66$])$

$$
S=\sum_{n \leqslant N} n^{i t}, \quad S=\sum_{a<n \leqslant b} n^{i t} .
$$

Здесь $t-$ растущий параметр, $t \geqslant t_{1}>0 ; N=N(t) \leqslant t$, или, часто, $N \leqslant \sqrt{t}$; точно также, $0<a<b \leqslant t$ или $b \leqslant \sqrt{t}$. Количество слагаемых в $S$ называется длиной cyммы, и чем больше в ней слагаемых, тем “более длинной” она является. Суммы $S$ и верхние оценки $|S|$ тесно связаны с порядком роста $|\zeta(\sigma+i t)|, t \rightarrow+\infty, \sigma-$ фиксированное число (см. [2, с. 112]). Естественно поставить вопрос о равномерных по $t$ и $N$ оценках дзетовых сумм и омега-теоремах для них. Похожие вопросы, но только для полных рациональных тригонометрических сумм, рассматривались автором в [3]. Отмечу также, что в [4] автор доказал омега-теорему для остаточного члена в многомерной проблеме делителей Дирихле, причем форма остаточного члена имеет такой вид, которьй был получен Дирихле, т.е. равномерньй по количеству слагаемых в сумме и количеству сомножителей, определяющих функцию делителей. Эта омега-теорема также связана с дзетовьми суммами.

Осреднение по $t$ квадрата модуля $S$ показывает, что “основное количество" $|S|$ оценивается сверху чуть более грубо, чем корень квадратньй из числа слагаемых. Действительно, если $T \geqslant T_{1}>0,2 \leqslant N \leqslant \sqrt{T}$ и множество $E \subset(T, 2 T)$ есть множество тех чисел $t$, для которых вьполняется неравенство

$$
\left|\sum_{n \leqslant N} n^{i t}\right| \geqslant \sqrt{N} h(T), \quad h(T)>0,
$$


то легко находим, что

$$
N h^{2}(T) \mu(E) \leqslant \int_{E}\left|\sum_{n \leqslant N} n^{i t}\right|^{2} d t \leqslant \int_{T}^{2 T}\left|\sum_{n \leqslant N} n^{i t}\right|^{2} d t \leqslant T N+16 N \log N \leqslant 17 T N
$$

Отсюда для меры $\mu(E)$ получаем оценку

$$
\mu(E) \leqslant T \Delta, \quad \Delta=17 h^{-2}(T) .
$$

Если взять $h(T)=\log ^{c} T, c>0$, то получаем, что для всех $t \in(T, 2 T)$, за исключением $t \in E \subset(T, 2 T), \mu(E) \leqslant 17 T \log ^{-2 c} T$, вьполняется оценка

$$
\left|\sum_{n \leqslant N} n^{i t}\right| \leqslant \sqrt{N} \log ^{c} T
$$

Ниже будет показано, что существуют бесконечная последовательность чисел $T$, $T \rightarrow+\infty$, и бесконечная последовательность чисел $N \leqslant \sqrt{T}, N \rightarrow+\infty$, такие, что нижняя оценка отвечающих им дзетовых сумм много больше, чем правая часть (1). Для доказательства теоремы 1 нужна будет нижняя оценка $|\zeta(1 / 2+i t)|$. Сформулирую в виде леммы 1 только частньй случай более общего утверждения из [5, с. 46].

Лемма 1. Существует постоянная $T_{0}>0$ такая, что при $T \geqslant T_{0}$ выполняется следуюшее неравенство:

$$
\max _{T \leqslant t \leqslant 2 T}\left|\zeta\left(\frac{1}{2}+i t\right)\right| \geqslant \exp \left(\frac{3}{4} \sqrt{\frac{\log T}{\log \log T}}\right)
$$

Теорема 1. Существует бесконечная последовательность чисел $t_{\nu}, \nu=1$, $2, \ldots$, таких, что $t_{1} \geqslant 1, t_{\nu+1} t_{\nu}^{-1} \geqslant 2$, и обладающих следующим свойством: $п р и$ каждом $t=t_{\nu}$ на интервале $(1, P), P=[\sqrt{t /(2 \pi)}]$, найдется по крайней мере $K$ натуральных чисел $N$,

$$
K \geqslant f^{2}(t), \quad \text { əдe } \quad f(t)=\exp \left(\frac{3}{5} \sqrt{\frac{\log t}{\log \log t}}\right)
$$

таких, что выполняется неравенство

$$
\left|\sum_{n \leqslant N} n^{i t}\right| \geqslant \sqrt{N} f(t)
$$


ДокаЗАТЕЛЬСтво. Возьмем в лемме $1 T=4^{\nu} T_{0}, \nu=1,2, \ldots$ Последовательность чисел $t_{\nu}$, существование которой утверждается в теореме, определяется равенством

$$
\max _{T \leqslant t \leqslant 2 T}\left|\zeta\left(\frac{1}{2}+i t\right)\right|=\left|\zeta\left(\frac{1}{2}+i t_{\nu}\right)\right| .
$$

Очевидно, что $t_{\nu+1} t_{\nu}^{-1} \geqslant 2, \nu=1,2, \ldots$. Положим, далее, $t=t_{\nu}$. Пусть при каждом натуральном числе $N, 1 \leqslant N \leqslant P$, вьполняется неравенство

$$
\left|\sum_{n \leqslant N} n^{i t}\right| \leqslant 2 \sqrt{N} f(t)
$$

Пользуясь приближенным уравнением Харди-Литтлвуда, находим

$$
\left|\zeta\left(\frac{1}{2}+i t\right)\right| \leqslant 2\left|\sum_{n \leqslant P} \frac{1}{\sqrt{n}} n^{i t}\right|+O\left(t^{-1 / 4}\right) .
$$

Полагая

$$
S(n)=\sum_{m \leqslant n} m^{i t},
$$

получаем

$$
\sum_{n \leqslant P} \frac{1}{\sqrt{n}} n^{i t}=\frac{1}{\sqrt{P}} S(P)+\sum_{1<n \leqslant P}\left(\frac{1}{\sqrt{n-1}}-\frac{1}{\sqrt{n}}\right) S(n-1) .
$$

Отсюда и из (2) следуют такие оценки:

$$
\begin{gathered}
\sum_{n \leqslant P} \frac{1}{\sqrt{n}} n^{i t} \leqslant 2 f(t)+\sum_{1<n \leqslant P} \frac{1}{n} 2 f(t)<2 f(t) \log t, \\
\left|\zeta\left(\frac{1}{2}+i t\right)\right| \leqslant 5 f(t) \log t, \quad t \geqslant t_{0}>0 .
\end{gathered}
$$

Но последнее неравенство противоречит оценке леммы 1. Поэтому предположение о том, что при каждом $N, 1 \leqslant N \leqslant P$, вьполняется (2), неверно: найдутся $N$ такие, что

$$
\left|\sum_{n \leqslant N} n^{i t}\right|>2 \sqrt{N} f(t)
$$

Пусть $N$ - одно из них. Из верхней тривиальной оценки $|S(N)|$ получаем

$$
N>2 \sqrt{N} f(t), \quad N>4 f^{2}(t) .
$$

Далее, при любом целом числе $M \geqslant 0$ справедливо равенство

$$
S(N+M)=S(N)+\sum_{N<n \leqslant N+M} n^{i t} .
$$

Переходя к неравенствам, будем иметь

$$
|S(N+M)| \geqslant|S(N)|-M>2 \sqrt{N} f(t)-M \geqslant \sqrt{N+M} f(t),
$$


если только $M \leqslant \sqrt{N} f(t) / 2$. Вспоминая, что $N>4 f^{2}(t)$, заключаем: существует по крайней мере $K, K \geqslant f^{2}(t)$, чисел $n$ таких, что

$$
|S(n)| \geqslant \sqrt{n} f(t)
$$

Теорема доказана.

Из теоремы 1 следует, что, по-видимому, нельзя получить оценку дзетовой суммы лучшую, чем, например, такая:

$$
|S(N)| \leqslant \sqrt{N} \exp \left(c \sqrt{\frac{\log t}{\log \log t}}\right),
$$

где $c>0$ - абсолютная постоянная.

Верхние оценки для $|S(N)|$, которые получаются даже из гипотезы Римана (будем назьвать ее RH), сильно отличаются от (3).

ТЕОРема 2. При справедливости RH имеет место следующая оценка:

$$
|S(N)| \leqslant \sqrt{N} \exp \left(c \frac{\log t}{\log \log t}\right)
$$

әде с $>0$ - абсолютная постоянная, $N \leqslant t$.

ДокАЗАТЕльство теоремы повторяет доказательство теоремы 1 из [6, с. 153] (или решение задачи 1 из [2, с. 112]) с использованием оценки $|\zeta(1 / 2+i t)|, t \geqslant t_{1}>0$, которая следует из RH (см., например, [7, с. 350]):

$$
\left|\zeta\left(\frac{1}{2}+i t\right)\right| \ll \exp \left(A \frac{\log t}{\log \log t}\right), \quad A>0 .
$$

Оценки дзетовых сумм (3) и (4) будут нетривиальньми, если вьполнены соответственно неравенства

$$
N \geqslant \exp \left(c_{1} \sqrt{\frac{\log t}{\log \log t}}\right), \quad N \geqslant \exp \left(c_{1} \frac{\log t}{\log \log t}\right),
$$

где $c_{1}>0$ - абсолютная постоянная, $t \geqslant t_{1}>0$. Вспоминая оценку $|S(N)|$, полученную методом Виноградова (см. [8] или $[1$, с. 95]):

$$
|S(N)| \leqslant N \exp \left(-c \frac{\log ^{3} N}{\log ^{2} t}\right)
$$

видим, что она будет нетривиальной, если только

$$
N \geqslant \exp \left(c_{1} \log ^{2 / 3} t\right)
$$

Следовательно, метод Виноградова позволяет оценивать нетривиально более короткие дзетовые суммы, чем те, которые оценены в теореме 2 на основе RH. Но оценки коротких дзетовых сумм позволяют уточнять границу нулей $\zeta(s)$. Посмотрим, что в этом случае дает оценка (3). Для этого нужна следующая лемма 2 (см. [7, с. 62]), устанавливающая связь между ростом $|\zeta(s)|$ и гранищей нулей $\zeta(s)$. 
Лемма 2. Пусть прu $t \rightarrow+\infty$

$$
\zeta(s)=O\left(e^{\varphi(t)}\right)
$$

в области

$$
1-\theta(t) \leqslant \sigma \leqslant 2, \quad t \geqslant t_{1}>0,
$$

где $\varphi(t)$ и $\theta^{-1}(t)$ - положительнье неубьвающие функиии такие, что $\theta(t) \leqslant 1$, $\varphi(t) \rightarrow+\infty, u$ кроме того,

$$
\frac{\varphi(t)}{\theta(t)}=o\left(e^{\varphi(t)}\right) .
$$

Тогда существует постоянная $A>0$ такая, что $\zeta(s)$ не имеет нулей в области

$$
\operatorname{Re} s=\sigma \geqslant 1-A \frac{\theta(2 t+1)}{\varphi(2 t+1)}, \quad t \geqslant t_{1}>0 .
$$

ТЕОРема 3. При выполнении оиенки (3) функиия $($ (s) не имеет нулей в области

$$
\operatorname{Re} s=\sigma \geqslant 1-c_{1} \sqrt{\frac{\log \log t}{\log t}}, \quad t \geqslant 14,
$$

где $c_{1}>0$ - абсолютная постоянная.

ДокАЗАТЕЛЬСТво. Из (3) и приближенного функционального уравнения $\zeta(s)$ находим оценку $|\zeta(\sigma+i t)|, 1 / 2 \leqslant \sigma \leqslant 1, t \geqslant 2$ :

$$
|\zeta(\sigma+i t)| \ll \exp \left(c_{2} \sqrt{\frac{\log t}{\log \log t}}\right), \quad c_{2}>0 .
$$

Полагая в лемме 2

$$
\varphi(t)=c_{2} \sqrt{\frac{\log t}{\log \log t}}, \quad \theta(t)=\frac{1}{2},
$$

приходим к утверждению теоремы.

Сравнивая (6) с границей нулей $\zeta(s)$, полученной методом Виноградова,

$$
\operatorname{Re} s=\sigma \geqslant 1-c_{1} \frac{1}{(\log t)^{2 / 3}(\log \log t)^{1 / 3}}, \quad t \geqslant 14,
$$

видим, что эти два результата не сильно отличаются один от другого.

Еше один вид оценок дзетовых сумм, частным случаем которого является уже рассмотренный вьше, вьглядит так:

$$
|S(N)| \leqslant N^{\beta} f(t),
$$

где $\beta$ - фиксированное число, $1 / 2 \leqslant \beta<1$. Пользуясь омега-теоремой Монтгомери (см. [9]):

$$
\zeta(\sigma+i t)=\Omega\left(\exp \left(c \frac{(\log t)^{1-\sigma}}{(\log \log t)^{\sigma}}\right)\right), \quad \frac{1}{2}<\sigma<1,
$$


и рассуждениями теоремы 1 , приходим к заключению, что, по-видимому, наилучшей возможной оценкой вида (7) может быть только такая, у которой

$$
f(t)=\exp \left(c \frac{(\log t)^{1-\beta}}{(\log \log t)^{\beta}}\right) .
$$

Повторяя рассуждения теоремы 3 , приходим к утверждению, что из (7) и (8) следует такая граница нулей $\zeta(s): \zeta(s) \neq 0$ в области $s$-плоскости вида

$$
\operatorname{Re} s=\sigma \geqslant 1-A \frac{(\log \log t)^{\beta}}{(\log t)^{1-\beta}}, \quad t \geqslant 14,
$$

$A>0$ - абсолютная постоянная.

Можно ставить вопрос об омега-оценках $|S(N)|$ другого вида, чем (7). Пусть при $1 \leqslant N \leqslant t$ вьполнена оценка

$$
|S(N)| \ll N \exp \left(-c \frac{\log ^{a} N}{\log ^{b} t}\right),
$$

где $c>0$ - постоянная, $a>b>0, t \geqslant t_{1}>0$. При $a=3, b=2$ такая оценка получена Виноградовым (см. [8], [1]). Пользуясь омега-оценкой $|\zeta(1+i t)|$ или теоремой Дирихле из теории диофантовых приближений, легко доказать, что

$$
\frac{a}{b} \ll \frac{\log \log t}{\log \log \log t}, \quad t \rightarrow+\infty .
$$

\section{СПИСОК ЦИТИРОВАННОЙ ЛИТЕРАТУРЫ}

[1] Карацуба А. А. Основы аналитической теории чисел. М.: Наука, 1975.

[2] Карацуба А. А. Основы аналитической теории чисел. 2-е изд. М.: Наука, 1983.

[3] Карацуба А. А. Об оценках полных тригонометрических сумм // Матем. заметки. 1967. T. 1. № 2. C. 199-208.

[4] Карацуба А. А. О связи многомерной проблемы делителей Дирихле с границей нулей $\zeta(s)$ // Матем. заметки. 2001. Т. 70. № 3. С. 477-480.

[5] Ramachandra K. On the Mean-Value and Omega-Theorems for the Riemann Zeta-Function. Bombay: Tata Inst. of Fund. Research, 1995.

[6] Воронин С. М., Карацуба А. А. Дзета-функция Римана. М.: Физматлит, 1994.

[7] Титчмарш Е. К. Теория дзета-функции Римана. М.: ИЛ, 1953.

[8] Виноградов И. М. Новая оценка функции $\zeta(1+i t)$ // Изв. АН СССР. Сер. Матем. 1958. T. 22. № 2. C. 161-164.

[9] Montgomery H. L. Extreme values of the Riemann zeta-function // Comment. Math. Helv. 1977. V. 52. P. 511-518. 